\title{
Arterial hypertension and telemedicine in times of pandemic
}

\begin{abstract}
The pandemic caused by the SARS-COV-2 virus started in late 2019 in China and spread rapidly around the world. The great impact it had on hypertensive patients has generated a change in the medical care paradigm, giving impetus to a virtual care modality, telemedicine. The objective of this work is to evaluate the technique of blood pressure measurement (equipment and procedure), its knowledge in this regard and, based on this, educate the hypertensive patient. Material and methods: descriptive and observational study of 426 patients, over 18 years of age, who requested a consultation by telemedicine in a community hospital, during July and November 2020. The patient was questioned about the equipment used, the measurement technique and on the knowledge of the blood pressure figures that defined him as hypertensive. Results: Of 426 patients, $49 \%$ were 62 -year-old women. $92.95 \%$ had a blood pressure monitor at home, of which $73 \%$ were electronic arm, $16.16 \%$ were electronic wrist and $10.84 \%$ manual. Only $53 \%$ had a validated blood pressure monitor. $35 \%$ of the total made at least 2 records and $39.2 \%$ knew their blood pressure values considered normal.
\end{abstract}

Volume |4 Issue 5 - 202|

\author{
Pronotti, María V,' Schiavone Miguel J,' Casas \\ Fernando, ${ }^{2}$ Medina Graciela, ${ }^{2}$ Saravia María \\ Angélica, ${ }^{2}$ Avaca Horacio ${ }^{2}$ \\ 'Department of Hypertension, Hospital Británico de Buenos \\ Aires (Hospital Británico), Argentina \\ ${ }^{2}$ Department of Cardiology, Hospital Británico de Buenos Aires \\ (Hospital Británico), Argentina
}

Correspondence: Pronotti, María V, Department of Hypertension, Hospital Británico de Buenos Aires (Hospital Británico),Argentina, Email vicki.pronotti@hotmail.com

Received: August 14,202I | Published: October 20, 202 I

Keywords: high blood pressure, SARS-COV-2 pandemic, telemedicine

\section{Introduction}

The pandemic outbreak of COVID-19 (produced by the SARSCov-2 virus) erupted in China on December 2019 and quickly spread around the world. Over 212 countries and territories have been and still are affected by the disease. In Argentina, the first case was reported on March 3rd 2020, and the first death by COVID-19 occurred on March 7th. On March 20th, quarantine was ordained at a national level.

COVID-19 has had an impact on patients with high blood pressure in several ways. In the first place, it has resulted in work termination and loss of health insurance, produced by economic decrease, physical inactivity resulting from mandatory lockdown, ordained quarantine, closure of gyms and recreation centers, and, in addition, has generateda change in medical attention for chronic disease, boosting attention through virtual methods, also known as telemedicine. In this context, self-measurement for arterial pressure (AP), which are random measurements performed by the patient, become of high importance to handle hypertension and cardiovascular failure prevention., ${ }^{1,2}$

Telemedicine is a technological tool not frequently used by Argentina's health providers, not only because there is a general lack of knowledge, but also due to the low access rate for the lower class population and the elderly groups, who have a steeper learning curve. However, this resource could improve health services, save resources, expand capacity for specialized attention in remote locations, ease clogged traditional medical services (emergencies or spontaneous demand) and is also a priceless instrument for education and research. The COVID-19 pandemic forces us to extend its usage and presents us with the opportunity to have a proper implementation. ${ }^{3}$

Such a tool is a complement to health services that must be regulated and integrated into the medical attention channels. It requires having up-to-date and monitored settings, clear security and confidentiality protocols, reliable and certified tele-diagnosis and telemonitoring software, resources for digital documentation storage and back-up, as well as support for education and research.
Considering this new format for care service, we as professionals wondered about the actual quality of blood pressure measurements, as reported by patients themselves. This concern arose in relation to the fact that such data would be the ground for health-decisions that would affect the aforementioned patients. The goal in our work was to evaluate the technique for blood pressure measurement (equipment and procedure), to understand the patients' knowledge on the matter and, based on such findings, to educate patients with high-blood patients on the proper behavior.

\section{Materials and methodology}

This work covers a cross-sectional, consecutive study that involved patients who were provided with a telemedicine appointment at a community hospital between July and December 2020. The appointments consisted of a questionnaire for the patient to respond about the equipment used for blood pressure control and measurement technique. Patients were also queried on which figures for blood pression would define a patient to be hypertensive. Patients included were at least 18 years old, and all of them had made appointments to consult on arterial hypertension.

For all cases, the following data was requested: The patient's age and gender; pressure meter available at their home or, if applicable, at the location where the measurement took place; which kind of pressure meter did patients had (manual or electronic). For electronic pressure meters, the brand and model was requested to evaluate its' validity. The interview also covered the technique for measurements, mostly related to the amount of measurements performed by the patient.

Lastly, patients were queried on figure thresholds that delimit arterial hypertension.

\section{Results}

The total amount of queried patients was $426,49 \%$ of which were women with an average age of 62 years old (Figure 1). 


\section{Women Men}

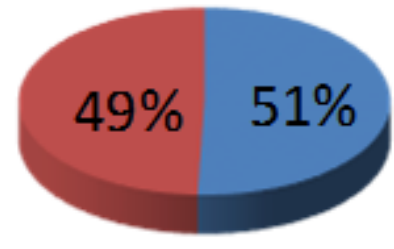

Figure I Percentage of patients surveyed by gender.

A $92.95 \%$ (396 patients) had blood pressure equipment at home, out of which $73 \%$ (289 patients) had electronic arm gadgets and $16.16 \%$ (64 patients) had electronic wrist gadgets. The remaining $10.84 \%$ (43 patients) had manual blood pressure equipment (Figure $2 \& 3)$.

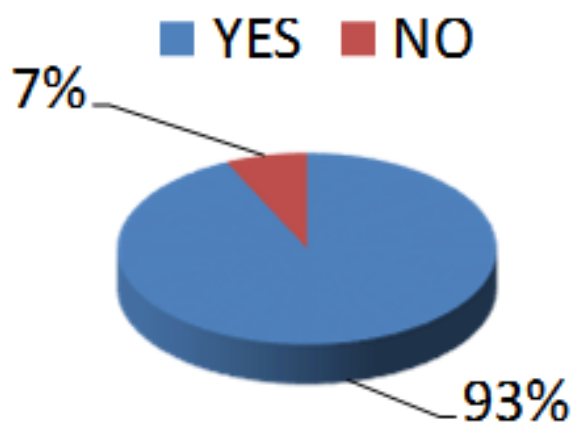

Figure 2 Percentage of patients with a pressure meter.

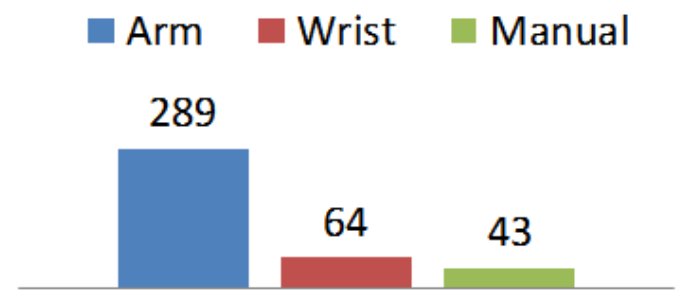

\section{Kinds of equipment}

Figure 3 Kinds of pressure meters owned by patients.

Out of all surveyed patients, only 53\% (210 patients) had a validated blood pressure equipment (Figure 4).

Lastly, knowledge about the disease was evaluated through the answered threshold value to determine arterial hypertension (taking into account that the expected values for at-home normal measurements were figures below $135 \mathrm{mmHg}$ for systolic blood pressure and/or $85 \mathrm{mmHg}$ for diastolic blood pressure) (Figure $4 \&$ 5). Only $39,2 \%$ (167 patients) were in the know about these expected values (Figure 6).

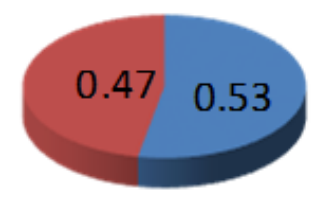

\section{Validated Not Validated}

Figure 4 Percentage of patients with validated equipment.

Regarding the technique in use for blood pressure measurement, only $35 \%$ (149 patients) performed at least 2 measurements (Figure 5).

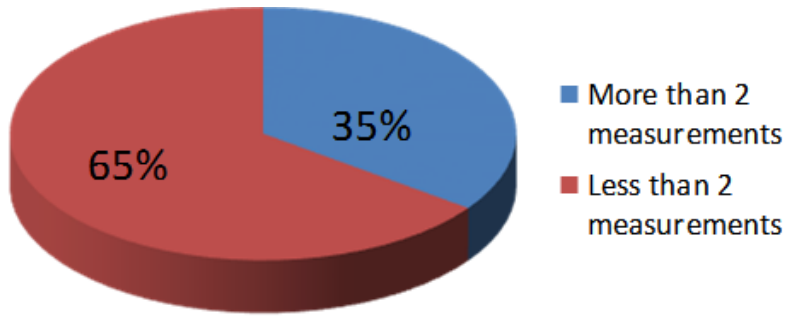

Figure 5 Applied technique for blood pressure measurement.

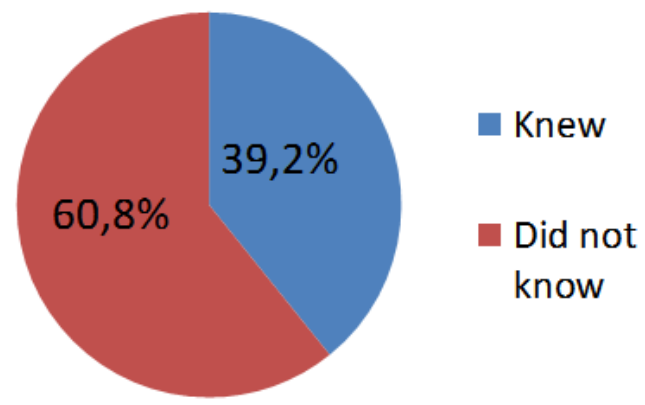

Figure 6 Percentage of patients that knew the normal figures for blood pressure.

\section{Discussion}

There is sufficient evidence on the impact of blood pressure as a risk factor and how its adequate treatment helps to reduce risks for cardiac and cerebrovascular events in the medium- and long-term. ${ }^{4-5}$ Measurements in addition to those performed by physicians have become a useful aid for this. This procedure eliminates the alarm reaction and is more reproductible; it presents a greater correlation with end-organ damage and its regression; also a greater correlation with cardiovascular events within a population in general and within hypertensive patients who have or have not received treatment; and it better evaluates, in a more precise way, how much blood pressure has receeded after treatment.

The PAMELA study, which took place in 2005, concluded that blood pressure in a medical facility is less correlated to total mortality reduction and to cardiovascular mortality. Home blood pressure measured (HBPM) followed in terms of usefulness for the same matter, while ambulatory blood pressure measurements were correlated the most with the aforementioned events (ABPM). ${ }^{6}$

Medical guides recommend self-measurement of blood pressure as a way to improve control and treatment adherence. ${ }^{2-9}$ There is evidence on this kind of measurement diminishing blood pressure by the 6th and 12th month, regardless of any interventions occurring in the middle. ${ }^{10}$ The technique is simple, quick and has a low cost. The goal is to better classify patients, increase percentages for positive controls and avoid mistakes in blood pressure measurements that might result in over or underestimating actual figures, leading to make mistaken decisions.

It is of most importance to apply a proper technique, and there is a minimal set of concepts the patient must acquire so as to ensure a reliable measurement. It is also of interest for patients to know about validated equipment and normal thresholds for blood pressure. For this reason, medical guides and the Argentinian Consensus for Arterial Hypertension SAC/FAC/SAHA (2018) suggest a ABPM measurement or home-monitoring (HBPM) to perform an accurate initial diagnosis and an eventual treatment update, given that such procedures, unlike self-monitoring, involve a validated piece of equipment and a technique with specific application protocols. ${ }^{11}$ 
This observational study demonstrates that not many patients own validated pressure meters; also, most of the subjects do not apply a proper technique and the understanding rate on normal thresholds for hypertension is low.

It is to be concluded that blood pressure self-measurement provides clear evidence of it, but in order to make medical decisions, it is important that doctors educate their patients. The current pandemic brought the many gaps on the matter to the front, making a point about most of the recorded measurements have a similar quality to those of self-monitoring than to those expected out of a blood pressure homemonitoring.

\section{Conclusion}

The ongoing pandemic forced us to find new tools for medical assistance. When it comes to hypertension, one of the most important goals is the proper blood pressure control through monitoring blood pressure, regardless of the procedure involved (self-monitoring, ABPM or HBPM). It is to be considered that not all patients have the socioeconomic means to perform ABPM or HBPM. In any case, everyone should have the same access to education and blood pressure monitoring within a medical facility, being that a physical or virtual appointment. This last option could resolve the accessibility barrier. However, the study raised attention on the low amount of validated equipment, the lack of knowledge around measurement technique for blood pressure and also around the figures that could be expected for such measurements. For this reason, it is of high importance for a physician to enhance communication and educate the patient on the matter.

\section{Acknowledgments}

None.

\section{Conflicts of interest}

No conflict of interest.

\section{Funding}

None.

\section{References}

1. https://www.conicet.gov.ar/wp-content/uploads/Informe_Final_CovidCs.Sociales-1.pdf

2. https://millionhearts.hhs.gov/index.html

3. Roldán-Gómez FJ, Jordán-Ríos A, Alvarez-Sangabriel A, et al. Telemedicina como instrumento de consulta cardiológica durante la pandemia COVID-19. Arch Cardiol Mex. 2020;90(Supl):88-93.

4. World Health Organization (WHO). Global atlas on cardiovascular disease prevention and control. Geneva, Switzerland: World Health Organization (WHO); 2011.

5. Ezzati M, Riboli E. Behavioral and dietary risk factors for noncommunicable diseases. $N$ Engl J Med. 2013;369(10):954-964.

6. Bombelli M, Toso E, Peronio M, et al. The Pamela Study: Main Findings and Perspectives. Curr Hypertens Rep. 2013;15(3):238-243.

7. Chobanian AV, Bakris GL, Black HR, et al. The Seventh Report of the Joint National Committee on Prevention, Evaluation, and Treatment of High Blood Pressure: the JNC7 report. JAMA. 2003;289(19):2560-2572.

8. Arnett DK, Blumenthal RS, Albert MA, et al. 2019 ACC/AHA guideline on the primary prevention of cardiovascular disease: a report of the American College of Cardiology/American heart association Task Force on clinical practice guidelines. Circulation. 2019;140(11):e596-e646.

9. Ferdinand KC, Vo TN, Echols MR. State-of-the-Art review: Hypertension practice guidelines in the era of COVID-19. Am J Prev Cardiol. 2020;2:100038.

10. Uhlig K, Patel K, Stanley IP, et al. Self-Measured Blood Pressure Monitoring in the Management of Hypertension A Systematic Review and Meta-analysis. Ann Intern Med. 2013;159(3):185-194.

11. Marcos M, Mario B, Paéz Olga y colaboradores. Consenso Argentino de Hipertensión Arterial. Revista Argentina de Cardiología. 86(supl 2):2018. 